\title{
Cervical, Trunk, and Lower Extremity Muscle Activities during Bridging Exercise on Stable vs. Unstable Bases of Support
}

\author{
Youngsang Ryu, MD, MSc ${ }^{1)}$, Hyolyun Roh, PhD, PT ${ }^{2)}$ \\ 1) Section of Tuina, Hospital to Liaoning University of Traditional Chinese Medicine \\ 2) Department of Occupational Therapy, Kangwon National University: Hwango-ri, Samcheok-si, \\ Gangwondo, 240-907 Republic of Korea. \\ TEL: +82 33-540-3481,FAX: +82 33-540-3489,E-mail: withtry@kangwon.ac.kr
}

\begin{abstract}
Purpose] The purpose of this study was to investigate the changes in the muscle activities of the neck, trunk, and lower extremities when an unstable base of support and a stable base of support were used in bridging exercises. [Subjects] The subjects of this study were 14 persons in their twenties ( 9 Males, 5 Females, 23.3 years old, $168.7 \mathrm{~cm}$, and $70.0 \mathrm{~kg}$ ). [Methods] The subjects performed an ordinary bridging position (stable base) and a bridging position with a dynamic ball cushion (unstable base). The muscular activities of the longissimus capitis and sternocleidomastoid in the neck, the erector spinae and rectus abdominis in the trunk, the rectus femoris and lateral hamstring in the thigh, and the tibialis anterior and lateral head of the gastrocnemius in the lower leg were measured using surface electromyography. [Results] The erector spinae, lateral hamstring and gastrocnemius activities were significantly higher on the unstable base of support than on the stable one. [Conclusion] Activity of muscle groups varies according to the stability of the base of support during bridging exercises. Therefore, conducting bridging exercises with lumbar pain patients requires a gradual change from a stable to an unstable base of support.

Key words: Base of support, Bridging exercise, Muscle activity
\end{abstract}

(This article was submitted Jan. 12, 2012, and was accepted Feb. 22, 2012)

\section{INTRODUCTION}

Lower back pain (LBP) patients have weakened deep muscles as compared to normal people, and their lack of reposition sense, resulting from reduced proprioceptive sense, may lead to problems with spinal stability, possibly causing recurrence of LBP $\rrbracket$. Proprioceptive signals are neural signals delivered from the mechanoreceptors located in the joints, articular capsules, ligaments, muscles, tendons, and skin to the central nervous system. Among the mechanoreceptors, the muscle receptors are the most important part in sophisticated limb position sense, and changes in the functional state of the muscles in relation to the role of muscle receptors may affect the sensitivity of the position sensef. Additionally, movement on an unstable surface, which triggers greater activities than movement on a stable

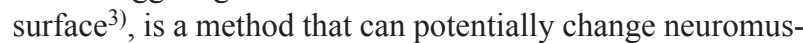
cular recruitment patterns. Even small changes in the soles in contact with the surface change the sensory input to the joint receptors and muscle receptors, and these changes of sensory input to the receptors induce postural reactions and affect the activity of nerves and muscles $\$$.

Bridging exercises are widely used in trunk stabilization program for LBP patients. The bridging exercise is a closedchain exercise, and it is an important motion for weight loading on the feet and knees in standing. It is useful for developing the capability to control sit to stand movements and facilitating pelvic motions. It also strengthens the lower part of the spine and the hip extensor muscles 1 . Moreover, bridging exercise retrains global muscles and local muscles to coordinate in the proper proportions in order to stabilize the trunk and the head when the arms or legs begin to move or are moving . In addition to inducing proper coordination between the global and local muscles, this exercise also contracts intrinsic muscles, such as the multifidus muscles, and superficial muscles, such as the abdominal muscles. Therefore, appropriate proprioceptive stimuli during a bridging exercise should efficiently enhance muscle activation and the ability to perform stabilization exercises. Accordingly, this study examined the changes in the muscle activities of the neck, trunk, and lower extremities when an unstable base of support, a dynamic air cushion, and a stable base of support were used in the bridging exercises.

\section{SUBJECTS AND METHODS}

The subjects of this study were 14 undergraduates (9 males, 5 females) at $\mathrm{G}$ University. They voluntarily consented to participate in this experiment. Their average ages, heights, and weights were $23.3 \pm 3.74$ years old, $168.7 \pm 5.10 \mathrm{~cm}$, and $70.0 \pm 15.21 \mathrm{~kg}$, respectively. The subjects were informed about the procedure and risks of the experiment and provided their written consent before participation. Subjects were selected if they had no neurological 

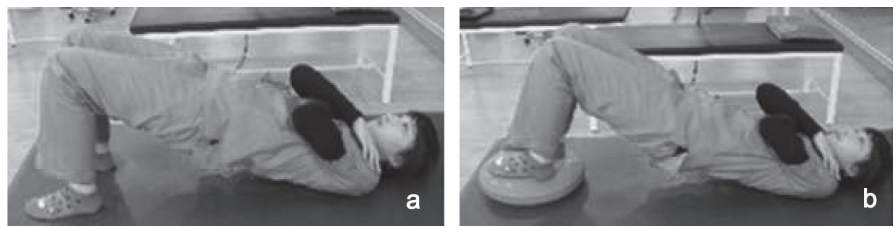

Fig. 1. a: Bridging exercise on the stable base, b: Bridging exercise on the unstable base

diseases, previous surgeries, or back pain within the last six months. Subjects were rejected if they had any abnormalities of the nervous system, cardiopulmonary system, or musculoskeletal system in the back or lower limbs.

The subjects performed an ordinary bridging position (method 1: stable base) and a bridging position using a dynamic ball cushion (method 2: unstable base). In bridging exercise method 1, the subject adopted the supine position on a bed, gathered both arms to the chest, dropped the knee joints off the side of the bed and bent them to 90 degrees while keeping the soles in contact with the ground. The subject then lifted the pelvis until hip joint flexion reached 0 degrees and maintained the position. In bridging exercise method 2 , the subject maintained the same position as that of method 1 with a Dynair Ball Cushion (TOGU, Germany) placed beneath the soles (Fig. 1). To familiarize themselves with the methods before performance of the bridging exercise, the subjects were given an explanation of the exercise methodology, and the exercises were then performed randomly using a random number table. One minute of rest was given after each of the exercises, because the exercises cause significant fatigue. The bridging position in each exercise was held for six seconds and the muscle activities of the last five seconds, ignoring the first one second, were used for data analysis.

In order to examine the flexor and extensor muscle activities of the trunk and lower extremities during each exercise, 8-channel wireless surface electromyography (EMG) (TeleMyo TM 2400T G2, Noraxon, USA) was employed, and $\mathrm{Ag}-\mathrm{Ag} / \mathrm{Cl}$ electrodes (Biopac, diameter $2 \mathrm{~cm}$ ) were used as electrodes. The EMG signals were sampled at a rate of $1000 \mathrm{~Hz}$, then full-wave rectified. For data storage, they were band-pass filtered at $60-500 \mathrm{~Hz}$ using MyoResearch XP Master Edition 1.06.64 XP software (Noraxon, USA), and noise was eliminated using a $60 \mathrm{~Hz}$ notch filter. For the analysis of the collected data, the five seconds of data were normalized and compared. A distance of $2 \mathrm{~cm}$ was maintained between the two electrodes and their potential differences were compared. The middle area of the belly was palpated and the electrodes were attached in parallel with the muscle fiber. In order to minimize the skin resistance, the area for electrode attachment was shaved and cleaned with alcohol. After the alcohol had dried completely, electrode gel was applied to the electrodes, which were then attached to the skin. The ground electrode was placed on the back of the right hand.

The muscles measured included the longissimus capitis and sternocleidmastoid in the neck, the erector spinae and rectus abdominis in the trunk, the rectus femoris and lateral hamstring in the thigh, and the tibialis anterior and lateral head of the gastrocnemius in the lower leg ${ }^{7-9}$. Surface electrodes were attached on the middle area between the cervical spinous process (C4) and the lateral surface of the trunk for the longissimus capitis; on the middle area of the muscle belly between the manubrium of the stern and the mastoid process for the sternocleidmastoid 10 ; ; on the middle area between the spinous process of the first lumbar vertebra (L1) and the lateral surface of the trunk for the erector spinaef; on the middle of the muscle belly between the navel and pubis for the rectus abdominis \$; on the anterior part of the femur, on the middle of the upper end of the patella and anterior superior iliac spine for the rectus femoris; on the posterior part of the femur, on the middle area between the ischial tuberosity and the fibular head for the lateral hamstring ${ }^{11}$; on the upper $30 \%$ area of the line connecting the knee joint lateral epicondyle and the calcaneus for the lateral head of gastrocnemius; and on the upper $75 \%$ area of the line connecting the knee joint lateral epicondyle and the ankle joint lateral malleolus for the tibialis anterior ${ }^{17}$.

In order to measure muscle activities, each exercise was measured for five seconds, and in order to standardize the measured values, the root mean square values of the raw data were calculated. To obtain mean values for the five-second measurements, each five-second motion was conducted a total of three times. To measure the mean values of the muscle activities, the first one second and the last one second were excluded and the remaining three seconds of data were used. The muscular activity was compared and analyzed with the mean of the measured values obtained from the three repeated measurements. In order to standardize the measurements of surface EMG, maximal voluntary isometric contraction (MVIC) values were established as baseline values, then each measurement was presented as the rate of maximal back muscle strength. Each individual subject had a different skin resistance, therefore, the measurements were subject to the above standardization process. The measurements were normalized to MVIC in order to produce standardized values for each individual subject. For the analysis of the measured data, the SPSS 12.0 Windows program was used, and the independent t-test was conducted to compare the two exercises. A value of $p<0.05$ was considered statistically significant.

\section{RESULTS}

There were no significant differences in the activities of 
Table 1. Comparison of muscle activities induced by bridging exercises on different bases of support

$(\mathrm{M} \pm \mathrm{SD})$ (unit: \%)

\begin{tabular}{clrc}
\hline Region & Muscle & \multicolumn{1}{c}{ stable } & \multicolumn{1}{c}{ unstable } \\
\hline \multirow{2}{*}{ Cervical } & Longissimus capitis & $5.64 \pm 6.07$ & $6.74 \pm 6.04$ \\
& Sternocleidomastoideus & $12.46 \pm 8.98$ & $13.76 \pm 7.79$ \\
\hline \multirow{2}{*}{ Trunk } & Rectus abdominis & $1.71 \pm 0.81$ & $1.67 \pm 0.69$ \\
& Erector spinae* & $15.81 \pm 8.21$ & $21.27 \pm 8.06$ \\
\hline \multirow{2}{*}{ Thigh } & Rectus femoris & $1.91 \pm 0.53$ & $1.95 \pm 0.33$ \\
& Biceps femoris* & $19.25 \pm 9.24$ & $26.41 \pm 11.71$ \\
\hline \multirow{2}{*}{ Leg } & Tibialis anterior & $10.86 \pm 10.23$ & $10.73 \pm 9.25$ \\
& Gastrocnemius* & $7.25 \pm 5.69$ & $16.71 \pm 11.40$ \\
\hline \multirow{2}{*}{$\mathrm{p}<0.05$} & & &
\end{tabular}

the longissimus capitis and sternocleidomastoideus muscles in the neck between the different bases of support. The rectus abdominis in the trunk did not show a significant difference in muscle activities between the stable base of support and the unstable one, while the erector spinae showed significantly higher muscle activity on the unstable base of support $(p<0.05)$. There was no significant difference in the activity of the rectus femoris between the stable and the unstable base of support, but the lateral hamstring activity was significantly higher on the unstable base of support than on the stable one $(\mathrm{p}<0.05)$. The activity of the tibialis anterior in the lower leg did not significantly differ between the stable and the unstable base, but that of the gastrocnemius was significantly higher on the unstable base of support $(\mathrm{p}<0.05)$ (Table 1).

\section{DISCUSSION}

The purpose of this study was to examine the effects of stable vs. unstable bases of support on cervical, trunk, femoral, and lower extremity muscle activities during bridging exercises. By examining the activities of the muscles in each body part, using different bases of support during bridging exercises, this study sought to provide basic information for the application and adaptation of bridging exercises.

A bridging exercise is a closed-chain, weight-bearing exercise for the lower extremities and the application of this exercise involves movements of not only the hip joint muscles, but also all the other joints and muscles within the closed chain 12. In this study, the activities of the erector spinae in the trunk, the biceps femoris in the thigh, and the gastrocnemius in the lower leg were significantly higher on the unstable base of support than on the stable base of support, demonstrating changes in the movements of all the muscles in the trunk, thigh, and lower leg during bridging exercises. Changes in the activities of the neck muscles were not statistically significant, but their activities increased on the unstable base of support, suggesting that bridging exercises on an unstable base of support more greatly influence muscle activity. The results of Stevens et al. (2006) are in agreement with the results of this study. They noted noting that exercise on an unstable surface triggered greater activities than exercise on a stable surface, and that exercise on an unstable base of support enhanced the position sense of the joints. Thus far, bridging exercises have been used to improve gluteus maximus and hamstring muscle strength 12 . These muscles are located in the dorsal part of the body. In this study, the muscle activities of the erector spinae, biceps femoris, and gastrocnemius, that are also located in the dorsal part of the body, were notable, consistent with the results of Kisner and Colby (2002) ${ }^{13}$. Kim (2009) demonstrated that when a $65-\mathrm{cm}$-diameter ball was placed below the foot, the activity of the erector spinae was greater than that of the rectus abdominis, the same as the result of this study ${ }^{14}$. To sum up the results of previous research on bridging exercises and this study, a change in the stability of the base of support during bridging exercise elicits changes in muscle activity.

Global muscle activity results in spinal overload and lumbar pain in patients prone to lumbar pain 15 . Therefore, when conducting bridging exercises for lumbar pain patients, a gradual change from a stable to an unstable base of support is required. Further, muscle strength, muscle activity, and lumbar pain may vary according to age. Thus, our present results should not be generalized. The participants of this study were healthy subjects in their 20 s. Future research including middle-aged and elderly lumbar pain patients should be conducted.

\section{REFERENCES}

1) O'Sullivan PB, Burnett A, Floyd AN, et al.: Lumbar repositioning deficit in a specific low back pain population. Spine, 2003, 28: 1074-1079. Medline CrossRef

2) Chiang JH, Wu G: The influence of foam surfaces on biomechanical variables contributing to postural control. Gait Posture, 1997, 5: 239-245. [CrossRef

3) Hogervorst T, Brand RA: Mechanoreceptors in joint function. J Bone Joint Surg Am, 1998, 80: 1365-1378. Medline

4) Stevens VK, Bouche KG, Mahieu NN, et al.: Trunk muscle activity in healthy subjects during bridging stabilization exercises. BMC Musculoskelet Disord, 2006, 7: 75. Medline CrossRef

5) Franklin DW, Osu R, Burdet E, et al.: Adaptation to stable and unstable dynamics achieved by combined impedance control and inverse dynamics model. J Neurophysiol, 2003, 90: 3270-3282. [Medline CrossRef

6) Schunk MC: Electromyographic study of the peroneus longus muscle during bridging activities. Phys Ther, 1982, 62: 970-975. Medline]

7) O’Sullivan SB, Schmitz TJ: Physical Rehabilitation: Assessment and Treatment, 4th ed. Philadelphia: F. A. Davis, 2001, pp 645-678.

8) Stevens VK, Coorevits PL, Bouche KG, et al.: The influence of specific 
588 J. Phys. Ther. Sci. Vol. 24, No. 7, 2012

training on trunk muscle recruitment patterns in healthy subjects during stabilization exercises. Man Ther, 2007, 12: 271-279. Medline [CrossRef]

9) Vezina MJ, Hubley-Kozey CL: Muscle activation in therapeutic exercises to improve trunk stability. Arch Phys Med Rehabil, 2000, 81: 1370-1379.

\section{[Medline] CrossRef}

10) Kim GS: Biosignal Measurement and Analysis. Laxtha Technical Research Institute. 2007, pp 29-30.

11) Cram JR, Kasman GS, Holtz J: Introduction to surface electromyography. Maryland: Aspen Pub, 1988, pp 223-384.
12) Eils E, Rosenbaum A: A multi-station proprioceptive exercise program in patients with ankle instability. Med Sci Sports Exerc, 2001, 33: 1991-1998. [Medline] [CrossRef]

13) Kisner C, Colby LA: Therapeutic Excercise: Foundations and Techniques. Philadelphia: F.A. Davis, 2002, pp 523-545.

14) Kim MJ: Effect of bridging stabilization exercises on trunk muscles activity on and off a swiss ball. PTK, 2009, 16: 18-24.

15) Richardson C, Jull G, Hodges P, et al.: Therapeutic exercises for spinal segmental stabilization in low back pain: Scientific basis and clinical approach. London: Churchill livingstone, 1999, pp 295-225. 